\title{
Power Scaling for Cognitive Radio
}

\author{
Niels Hoven \\ nhoven@eecs.berkeley.edu \\ Anant Sahai \\ sahai@eecs.berkeley.edu \\ Dept. of Electrical Engineering and Computer Science \\ University of California, Berkeley
}

\begin{abstract}
In this paper we explore the idea of using cognitive radios to reuse locally unused spectrum for their own transmissions. We impose the constraint that they cannot generate unacceptable levels of interference to licensed systems on the same frequency. Using received SNR as a proxy for distance, we prove that a cognitive radio can vary its transmit power while maintaining a guarantee of service to primary users. We consider the aggregate interference caused by multiple cognitive radios and show that aggregation causes a change in the effective decay rate of the interference. We examine the effects of heterogeneous propagation path loss functions and justify the feasibility of multiple secondary users with dynamic transmit powers. Finally, we prove the fundamental constraint on a cognitive radio's transmit power is the minimum SNR it can detect and explore the effect of this power cap.
\end{abstract}

\section{INTRODUCTION}

Contrary to popular belief, actual measurements show that most of the allocated spectrum is vastly underutilized at any specific location and time [1]. As a result, the FCC's exclusiveuse spectrum allocation policy is being increasingly viewed as outdated. Proponents of unlicensed devices assert that "wireless transmissions can be regulated by a combination of (a) baseline rules that allow users to coordinate their use, to avoid interference-producing collisions, and to prevent, for the most part, congestion, by conforming to equipment manufacturers specifications, and (b) industry and government-sponsored standards" [2]. Recognizing the need for increased spectrum efficiency, a number of organizations such as New America, the Center for Digital Democracy, and Free Press, have already thrown their support behind spectrum deregulation [3].

However, further research is required to lay the groundwork for the technology that policy makers assume already exists. Cognitive radios are one proposed idea to scavenge this open spectrum [4], [5]. These radios would be designed to dynamically adapt their transmissions to their environment, though fundamental questions of the concept's practicality still remain open. First, can practical cognitive systems even operate without causing excessive interference to legacy users? Proving so is essential to convincing the FCC to open more spectrum to unlicensed devices. Second, can useful wireless systems operate under these constraints? In this paper we target the former issue, focusing on non-interference to the primary system rather than realizable benefits for the secondary systems. We show the existence of constraints that allow multiple cognitive radios to transmit at reasonable power levels while maintaining a guarantee of service to legacy/priority users on the same band. We do not consider achievable data rates or necessary protocols for the secondary systems.

A number of concerns must be alleviated before the FCC will allow cognitive radios into already allocated bands. We have already proved that practical cognitive radios must be able to detect the presence of undecodable signals [6]. To make matters worse, slight uncertainty in the noise causes serious limits in detectability [7]. These barriers can be overcome if the licensed (primary) transmitter transmits a perfectly known pilot signal or training sequence to aid detection. Coherent detection via a matched filter results in processing gain and more effective signal detection. Furthermore, pilots allow users to measure the local $\mathrm{SNR}^{1}$ of the primary signal, which can then be used as a proxy for distance from the primary transmitter. Armed with this information, cognitive radios (secondary users) can approximate their distance from the primary transmitter and adjust their transmit power accordingly.

In this paper we present an example of a power control rule which allows secondary users to aggressively increase their transmit powers while still guaranteeing an acceptable level of aggregate interference at the primary receivers. Of particular concern are the effect of different propagation path losses for different systems, the effect of multiple cognitive users, and the effect of heterogeneous transmit powers among the cognitive users. We demonstrate that none of these issues invalidates cognitive radio's feasibility.

After a brief review of previous work (II) and a description of our model (III), we consider a single secondary user sharing spectrum with the primary system. As an example, imagine a cognitive radio broadcasting his signal over underutilized television bands far outside a city. We further divide the single secondary user regime into two subcases. We can think of the secondary users as licensed users constrained to a specific power ${ }^{2}$. On the other hand, it seems reasonable to allow a secondary user to use more power if he is further from the primary system. We examine both these cases in section $\mathrm{V}$,

\footnotetext{
${ }^{1}$ This can be measured by allowing the primary transmitter's pilot signal to double as a synchronization signal so that the secondary transmitters can periodically all fall silent together and measure their local SNR without interference from other secondaries. Alternatively, SINR could be used if the interference resulting from other secondary users can be determined.

${ }^{2}$ This could happen if two distinct property rights were auctioned off for a particular frequency band. For example, company A could purchase the right to transmit up to $10 \mathrm{~kW}$ on the $800-900 \mathrm{MHz}$ band anywhere in the US. while company $\mathrm{B}$ could purchase the right to transmit up to $1 \mathrm{~kW}$ on the same band but only when A is not using it.
} 
followed by the effects of shadowing/fading in section VI. In section VII, we extend our analysis to multiple secondary users. We consider first the case in which all the secondary users are bound by the same power constraint. Finally, in section VIII, we allow the secondary users to be heterogeneous in nature and to increase their transmit power with distance from the primary system. This final viewpoint more closely aligns with the traditional view of cognitive radio [5].

\section{RELATED WORK}

The idea that interference is local and frequencies may be reused is not new. Spatial considerations for frequency reuse have been studied extensively in cellular systems [8], [9]. However, these systems differ from the cognitive radio case in a number of significant ways.

Most of the interference in a cellular system is withinsystem interference, caused by devices the spectrum owner designs. It can therefore be tightly controlled, both in terms of its power and its spectral characteristics.

Cognitive radios, on the other hand, do not just cause out-ofcell interference, they cause out-of-system interference. This interference comes from a sea of heterogeneous devices with varying powers, duty cycles, and even propagation path losses. Previous research into frequency reuse in cellular networks has made the reasonable assumption of user homogeneity [10], [11], [12]. When considering the interaction between cellular telephones or 802.11 access points, one can assume that incell and out-of-cell transmitters use the same power control rules and experience the same propagation path loss.

In the case of cognitive radios, however, these assumptions do not hold. Low-powered cognitive radios may be sharing spectrum with a tall television transmitter. We expect the cognitive radios will be operating at ground level and hence will experience faster signal attenuation [13].

This sea of heterogeneous secondary devices must be able to guarantee service to primary users who are already near the limit of decodability in the no outside-interference case. Cell planners, designing their cell sizes to guarantee an acceptable level of interference, often have the additional advantage of a network of base stations whose locations are fixed and known. For (potentially mobile) cognitive radios, which might lack any sort of regulated infrastructure, we will use locally measured SNR in place of distance.

\section{MODEL}

Some of the most promising bands for unlicensed devices are the TV broadcast bands. The FCC has already released a Notice Of Proposed Rule Making exploring the operation of unlicensed devices on spatially/temporally "unused" television broadcast bands [14].

In our model, we assume a band already potentially assigned to a high-powered single-transmitter system (television, for example). Within some protected radius of the primary transmitter, all unshadowed primary receivers must be guaranteed reception, even when the cognitive radios are operating. All transmissions are assumed to be omnidirectional.
The primary system has a minimum required SINR to successfully decode at its target rate $\mathrm{R}$. In the absence of interference, this $\gamma_{d e c}$ occurs at a radius $r_{d e c}$ from the transmitter. The idea is to guarantee service to primary users within some protected radius $\left(r_{p}\right)$ by defining an additional "no-talk radius" $\left(r_{n}\right)$ within which secondary users must be quiet (Figure 1). At distances from the primary transmitter greater than $r_{n}$, secondary users might be allowed to transmit. Ideally, these "no-talk regions" would be centered on each of the primary system's receivers, but we are assuming that the cognitive radios have no way of knowing these locations.

Since we are using locally measured SNR as a proxy for distance, it is convenient to represent $r_{d e c}, r_{p}$, and $r_{n}$ in terms of the SNR in $\mathrm{dB}$ measured at those points. However, if the primary receiver is a TV antenna on a roof, it might measure an SNR of $0 \mathrm{~dB}$ at one location, while a cognitive radio on the ground at the same location might measure $-10 \mathrm{~dB}$. Therefore we must specify who is measuring the SNR at each distance. We consider $\gamma_{d e c}$ and $\gamma_{p}$ to be measured by a primary receiver, while $\gamma_{n}$ is by a secondary transmitter. Denoting the power of the primary transmitter as $P_{1}$, and the power of the noise ${ }^{3}$ at the primary receiver $\sigma^{2}$, we define:

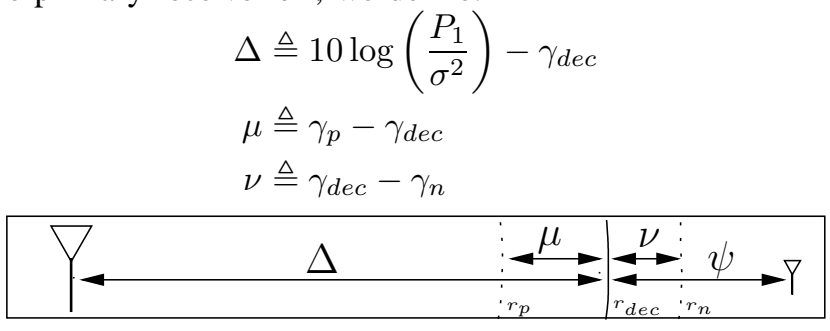

Fig. 1. SNR margins can be used as a proxy for distance

For example, if the minimum decodable SNR for the primary receiver is $10 \mathrm{~dB}$ and a secondary transmitter measures an SNR of $-5 \mathrm{~dB}$ at $r_{n}$, then $\nu=15$. We also define $\psi$ to be the margin between $\gamma_{d e c}$ and the local SNR measured by a particular secondary user.

As in [15], we represent the propagation-related power attenuation between two users a distance $r$ apart as a function $g(r)$ defined on $[0, \infty]$. We require $g(r)$ to be continuous with $0<g(r) \leq C \cdot r^{-2-\epsilon}$ (free-space propagation loss) for some $C>0, \epsilon>0$. We allow different gain functions $g_{11}(r)$, the propagation path loss between the primary transmitter and primary receiver, $g_{12}(r)$, between the primary transmitter and secondary receiver, and $g_{21}(r)$, between the secondary transmitter and primary receiver. Throughout this paper, our examples will be $g_{11}(r)=g_{12}(r)=r^{-\alpha_{1}}$ and $g_{21}(r)=r^{-\alpha_{2}}$.

For example, for the gain function $g_{11}(r)=r^{-3.5}, \Delta=165$ corresponds to a transmitter with about a $52 \mathrm{~km}$ range:

${ }^{3}$ If there is uncertainty in the noise power, then we can choose $r_{\text {dec }}$ first and set $\sigma^{2}$ to the maximum tolerable noise at that radius. If the noise power crosses this threshold with the cognitive radios transmitting, then protected users at $r_{p}$ could experience an outage. However, this noise event would cause an outage for primary users at $r_{d e c}$ even without the cognitive radios. We also assume that this $\sigma^{2}$ is preprogrammed into the cognitive radio, so it does not need to be continually estimated. Designing radios to compensate for changing noise floors is a topic for future research. 


$$
\begin{aligned}
\Delta & =165=10 \log \left(\frac{P_{1}}{\sigma^{2}}\right)-10 \log \left(\frac{P_{1} r_{\text {dec }}^{-4}}{\sigma^{2}}\right) \\
r_{\text {dec }} & =10^{\frac{165}{3.5 \cdot 10}} \approx 51800
\end{aligned}
$$

Further, consider $\mu=1 \mathrm{~dB}$ and $\nu=0.01 \mathrm{~dB}$ as potential operating margins. In this case, $r_{d e c}-r_{p} \approx 3300 \mathrm{~m}$, while $r_{n}-r_{\text {dec }} \approx 34 \mathrm{~m}$. Because of the large protected radius, very small increments in $\mathrm{dB}$ correspond to large physical distances. This is important, because it means that the necessary operating margins can be quite small. Unless otherwise specified, we will assume $\mu=1$ for our plots.

\section{OUT-OF-SYSTEM INTERFERENCE}

The interference from the secondary systems will be greatest to a user at the edge of the protected radius $r_{p}$ (Figure 2).

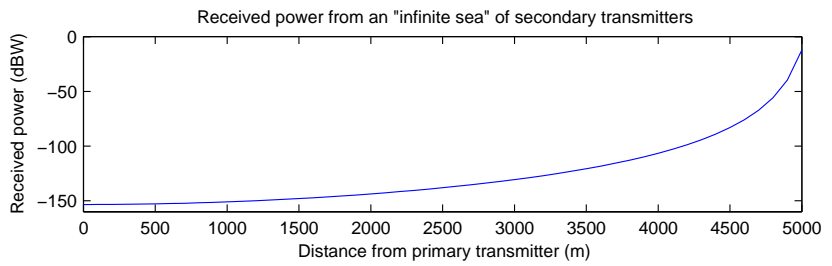

Fig. 2. Interference from secondary transmitters increases towards the border of the protected region. The secondary sea begins at $5100 \mathrm{~m}$, at which point the interference becomes infinite.

That primary user has a maximum amount of out-of-system interference that it can tolerate. We examine the maximum allowable power for a secondary system while still guaranteeing decodability (SINR $\geq \gamma_{d e c}$ ) to a user on the protected border. $Q_{1}$ and $Q_{2}$ denote the primary and aggregate secondary transmitters' powers at the primary receiver, i.e. $Q_{1}=P_{1} g_{11}\left(r_{p}\right)$ for a receiver on the edge of the protected region. A guarantee of reception can therefore be expressed as:

$$
\begin{aligned}
\frac{Q_{1}}{Q_{2}+\sigma^{2}} & \geq S I N R_{d e c}=10^{\frac{\gamma_{d e c}}{10}} \\
Q_{2} & \leq Q_{1} 10^{-\frac{\gamma_{d e c}}{10}}-\sigma^{2}
\end{aligned}
$$

We can express $Q_{1}$ on the protected border in terms of SNR:

$$
\begin{aligned}
\log \left(\frac{Q_{1}}{\sigma^{2}}\right) & =\gamma_{d e c}+\mu \\
Q_{1} & =\sigma^{2} 10^{\frac{\gamma_{d e c}+\mu}{10}}
\end{aligned}
$$

Substituting into equation (2), we see that the secondary system must guarantee:

$$
Q_{2} \leq\left(10^{\frac{\mu}{10}}-1\right) \sigma^{2}
$$

This is a fundamental constraint for any secondary system.

\section{SINGLE SECONDARY TRANSMITTER}

A one-size-fits-all power constraint must consider a primary receiver and secondary transmitter as close as possible, with the primary on the edge of the protected zone and the secondary on the edge of the no-talk zone. (4) implies:

$$
\begin{aligned}
P_{2} g_{21}\left(r_{n}-r_{p}\right) & \leq\left(10^{\frac{\mu}{10}}-1\right) \sigma^{2} \\
P_{2} & \leq\left(10^{\frac{\mu}{10}}-1\right) \sigma^{2}\left(g_{21}\left(r_{n}-r_{p}\right)\right)^{-1}
\end{aligned}
$$

A more interesting case occurs when the single secondary transmitter is allowed to vary its power depending on its proximity to the protected region. We simply replace the worst case distance $r_{n}$ in equation (5) by the secondary's actual distance $r_{2}$ from the primary transmitter. We observe that this new power schedule is strictly better than the "power licensed" scenario because a secondary user on the edge of the no-talk region is now a worst case scenario. Since these distances are not known to the cognitive radio, we perform our calculations in terms of SNR.

First, we write $r_{p}$, the protected radius, in terms of SNR.

$$
\begin{aligned}
10 \log \left(\frac{P_{1}}{\sigma^{2}}\right)-10 \log \left(\frac{P_{1} g_{11}\left(r_{p}\right)}{\sigma^{2}}\right) & =\Delta-\mu \\
g_{11}\left(r_{p}\right) & =10^{\frac{-\Delta+\mu}{10}} \\
r_{p} & =g_{11}^{-1}\left(10^{\frac{-\Delta+\mu}{10}}\right)
\end{aligned}
$$

Next, we solve for the distance $r_{2}$ of the secondary transmitter, in terms of his local SNR.

$$
\begin{aligned}
10 \log \left(\frac{P_{1}}{\sigma^{2}}\right)-10 \log \left(\frac{P_{1} g_{12}\left(r_{2}\right)}{\sigma^{2}}\right) & =\Delta+\psi \\
g_{12}\left(r_{2}\right) & =10^{\frac{-\Delta-\psi}{10}} \\
r_{2} & =g_{12}^{-1}\left(10^{\frac{-\Delta-\psi}{10}}\right)
\end{aligned}
$$

Equations (6) and (7) let us write (5), the maximum allowable power for a secondary transmitter, in SNR terms.

$$
\begin{aligned}
\frac{P_{2}}{\sigma^{2}} & \leq\left(10^{\frac{\mu}{10}}-1\right)\left(g_{12}\left(r_{2}-r_{p}\right)\right)^{-1} \\
& =\frac{\left(10^{\frac{\mu}{10}}-1\right)}{\left(g_{21}\left(g_{12}^{-1}\left(10^{\frac{-\Delta-\psi}{10}}\right)-g_{11}^{-1}\left(10^{\frac{-\Delta+\mu}{10}}\right)\right)\right)}
\end{aligned}
$$

For our example gain functions $g_{11}(r)=g_{12}(r)=r^{-\alpha_{1}}$ and $g_{21}(r)=r^{-\alpha_{2}}$ this gives us:

$$
\begin{aligned}
10 \log \left(\frac{P_{2}}{\sigma^{2}}\right) \leq & \frac{\alpha_{2}}{\alpha_{p}} \Delta+10 \log \left(10^{\frac{\mu}{10}}-1\right) \\
& +10 \alpha_{2} \log \left(\left(10^{\frac{\psi}{10}}\right)^{\frac{1}{\alpha_{p}}}-\left(10^{-\frac{\mu}{10}}\right)^{\frac{1}{\alpha_{p}}}\right)
\end{aligned}
$$

The first term describes how aggressively the primary user is transmitting, i.e. how far a user can travel from the primary transmitter and still decode the signal. Increasing the primary transmitter's rate without increasing its power decreases $\Delta$ and therefore requires the secondary transmitter to quiet down. The second term represents how tolerant the protected primary receivers are to interference. The final term represents how far the secondary transmitter is from the protected receivers. Also note that if $\psi=-\mu$ the secondary transmitter is in the protected region and must be silent.

Figure 3 shows the effects of the margin $\Delta$ between the primary transmitter and the decodability radius, and also the margin $\mu$ between the protected radius and the decodbility radius. We observe a few interesting effects.

First, assuming the cognitive radios wish to transmit at -10 $\mathrm{dbW}$, a small $\Delta$ presents a problem (Figure $3 \mathrm{a}, \mathrm{b}$ ). This requires a large margin $\psi$ (far in excess of $30 \mathrm{~dB}$ ). Secondary users must therefore be far more sensitive than the primary users. 
For small $\mu$ (Figure 3a,c), the maximum allowable power for the secondary transmitter jumps from zero to possibly healthendangering levels as soon as the secondary transmitter is outside the protected radius. If the secondary users are low powered devices then they only need to know when they are slightly outside the protected region. Therefore, the cognitive radio does not need to be significantly more sensitive than the users of the primary system, which are capable of receiving a signal all the way out to the decodability radius. Because the increase in allowable power is so rapid, any sensitivity beyond that hardly buys the secondary user anything.

A larger margin $\mu$ allows secondary transmitters to transmit geographically much closer to the protected region. Not only does this allow much greater reuse of physical space, but more powerful secondary transmitters (an $802.11 \mathrm{~g}$ access point is $\approx-10 \mathrm{dBW}$ ) can also have far less sensitive detectors (Figure 3d). This tradeoff cannot be resolved technically; a policy decision needs to be made.

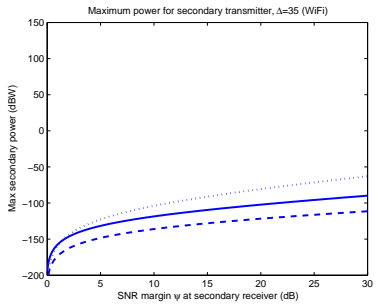

(a) $\Delta=35 \quad\left(r_{d e c}=10 \mathrm{~m}\right), \mu=0.1$

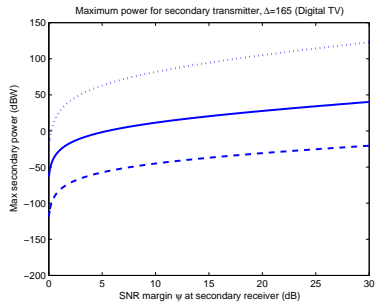

(c) $\Delta=165\left(r_{d e c}=52 \mathrm{~km}\right), \mu=0.1$

$$
\begin{array}{ll}
\cdots \cdots \cdots & \alpha_{1}=3.5, \alpha_{2}=5 \\
\hdashline \quad \alpha_{1}=3.5, \alpha_{2}=3.5 \\
-\_-\alpha_{1}=5, \alpha_{2}=3.5
\end{array}
$$

Fig. 3. Maximum power for a secondary transmitter vs. $\mathrm{dB}$ beyond $r_{d e c} . \Delta$ measures the primary signal attenuation from the transmitter to the decodability radius. For reference, we also give the distance to the decodability radius, assuming attentuation as $r^{-3.5}$ (i.e. $\alpha_{1}=3.5$ ).

The figures also illustrate the effect of different decay rates $\alpha_{1}$ and $\alpha_{2}$. If $\alpha_{2}>\alpha_{1}$, i.e. the secondary user's transmissions attenuate faster with distance than the primary transmissions do, we see the secondary user can use more power than he could if both systems experienced the same path loss. This is likely to be the case, for example, if the primary transmitter is a tall TV antenna while the secondary users are located on the ground. The significance of the case in which $\alpha_{1}>\alpha_{2}$ will become apparent in section VII.

\section{SingLE SECONDARY TRANSMITTERS WITH SHADOWING/FADING}

We now extend our model to the case in which the secondary transmitter may lie in a shadow (signal loss of $\beta \mathrm{dB}$ ) with respect to the primary signal. A secondary transmitter must now measure a margin of $\nu+\beta$ to be certain that he is outside the no-talk zone. Adjusting equation (9) to account for shadowing, we get:

$$
\frac{P_{2}}{\sigma^{2}} \leq \frac{\left(10^{\frac{\mu}{10}}-1\right)}{\left(g_{21}\left(g_{12}^{-1}\left(10^{\frac{-\Delta-(\psi-\beta)}{10}}\right)-g_{11}^{-1}\left(10^{\frac{-\Delta+\mu}{10}}\right)\right)\right)}
$$

Because a secondary transmitter can never assume it is unshadowed, we see that shadowing results in a pure shift of the curve in Figure 4. As an aside, we are assuming the gain functions are deterministic, but since we are using local SNR as our distance metric, this result can be extended to a dynamically fading channel by treating a multipath fade as additional shadowing [16]. However, one distiction is worth noting. While multipath fading can be nearly independent between nearby users, shadowing is likely to be highly correlated. In other words, future techniques to improve detection (such as cooperation among cognitive radios) may be more effective against multipath fading than against shadowing.

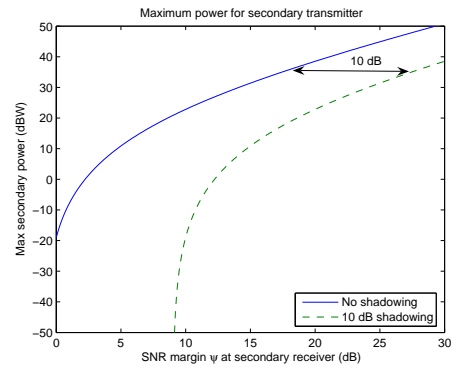

Fig. 4. The possibility of $10 \mathrm{~dB}$ of shadowing results in a $10 \mathrm{~dB}$ shift of the required SNR margin (eqn. 10).

\section{Multiple LiCEnSED SECONDARY TRANSMitTERS}

Suppose now that we are no longer limited to a single interferer. Outside of the no-talk circle of radius $r_{n}$, we assume there exists a sea of secondary transmitters, each with power $P_{2}$. We further assume that there is a limit to how densely these transmitters are packed. Each secondary transmitter uniquely occupies a footprint of area A, so this "secondary sea" has a power density $D=\frac{P_{2}}{A}$. Integrating over this sea gives the aggregate power of the secondary transmissions at a primary receiver on the edge of the protected region.

As in the case of the single licensed transmitter, we assume a constant power density outside the no-talk zone. Later, in section VIII, we will consider the case in which secondary users are allowed to increase the power of their transmissions as they venture further from the protected region.

We first assume that the secondary transmission power decays as $g_{21}(r)=r^{-\alpha_{2}}, \alpha_{2}>2$. We also assume $r_{p} \gg$ $r_{n}-r_{p}$, the distance between the primary receivers and the secondary transmitters. For a primary receiver on the edge of the protected region, the "coast" of the secondary sea can be 


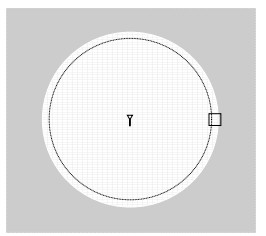

(a) Secondary sea

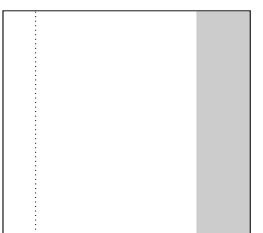

(b) Sea detail
Fig. 5. A circular coast looks like a straight line to primary users near the edge of the protected radius.

approximated by a line a distance of $r_{n}-r_{p}$ away. We examine the quality of this approximation in a later calculation (14).

$$
\begin{aligned}
Q_{2} & =\int_{-\frac{\pi}{2}}^{\frac{\pi}{2}} \int_{\frac{r_{n}-r_{p}}{\cos (\theta)}}^{\infty} D r^{-\alpha_{2}} r d r d \theta \\
& =\frac{D}{-\alpha_{2}+2} \int_{-\frac{\pi}{2}}^{\frac{\pi}{2}}\left[r^{-\alpha_{2}+2}\right]_{\frac{r_{n}-r_{p}}{\cos \theta}}^{\infty} d \theta \\
& =\frac{D \cdot\left(r_{n}-r_{p}\right)^{-\alpha_{2}+2}}{\alpha_{2}-2} \int_{-\frac{\pi}{2}}^{\frac{\pi}{2}}(\cos \theta)^{\alpha_{2}-2} d \theta \\
& =D \cdot K\left(\alpha_{2}\right) \cdot\left(r_{n}-r_{p}\right)^{-\alpha_{2}+2}
\end{aligned}
$$

where $K\left(\alpha_{2}\right)=\frac{\int_{-\frac{\pi}{2}}^{\frac{\pi}{2}}(\cos \theta)^{\alpha_{2}-2} d \theta}{\alpha_{2}-2}$.

For $\alpha_{2}=6, K\left(\alpha_{2}\right)=\frac{1}{4} \frac{\alpha_{2}-2}{4 ! !} \pi \approx 0.295$.

The entire sea of secondary transmitters behaves like a single transmitter of power $D \cdot K\left(\alpha_{2}\right)$, located a distance $r_{n}-r_{p}$ away (i.e. at the coast of the sea), but with a new decay exponent of $-\alpha_{2}+2$.

For small $r_{n}-r_{p}$, the majority of the interference comes from the few secondaries closest to the vulnerable primary receiver. As the secondary sea moves further away, more of the sea becomes "visible" to the receiver, explaining why the decay is now as $r^{-\alpha_{2}+2}$. The effect of increasing the secondary decay exponent by two is illustrated in Figure 3.

This approximation, however, understates the interference caused by the secondary sea in Figure 5a. We can upperbound the interference by considering the case where a primary receiver is completely surrounded by a secondary sea a distance $r_{n}-r_{p}$ away. Using our generalized gain functions:

$$
\begin{aligned}
Q_{2} & =\int_{-\pi}^{\pi} \int_{r_{n}-r_{p}}^{\infty} D g_{21}(r) r d r d \theta \\
& =D \int_{-\pi}^{\pi}\left[r \tilde{g}_{21}(r)-\check{g}_{21}(r)\right]_{r_{n}-r_{p}}^{\infty} d \theta
\end{aligned}
$$

where $g_{21}(r)=\frac{d}{d r} \tilde{g}_{21}(r)=\frac{d^{2}}{d r^{2}} \check{g}_{21}(r)$. Since $g(r) \leq C$. $r^{-2-\epsilon}, \lim _{r \rightarrow \infty}\left(r \tilde{g}_{21}(r)-\check{g}_{21}(r)\right)=0$ and it follows:

$$
Q_{2}=D \cdot 2 \pi \cdot\left(\check{g}_{21}\left(r_{n}-r_{p}\right)-\left(r_{n}-r_{p}\right) \tilde{g}_{21}\left(r_{n}-r_{p}\right)\right)
$$

Specifically, for our example gain functions, we have

$$
Q_{2}=D \cdot \frac{2 \pi}{\alpha_{2}-2} \cdot\left(r_{n}-r_{p}\right)^{-\alpha_{2}+2}
$$

Again, this bound claims that the sea behaves like a single transmitter of power located a distance $r_{n}-r_{p}$ away but with a new decay exponent $-\alpha_{2}+2$.
Figure 6 shows that the straight line approximation is quite good. Having multiple secondary users does change the decay rate of the aggregate interference, but we have already seen that the limits on the allowable transmit power can still be quite generous. For systems with smaller $\Delta$, the change in decay rate could be compensated for with either a larger SNR margin $\nu$ to the no-talk zone, or a larger SNR margin $\mu$ between the protected radius and the decodability limit.

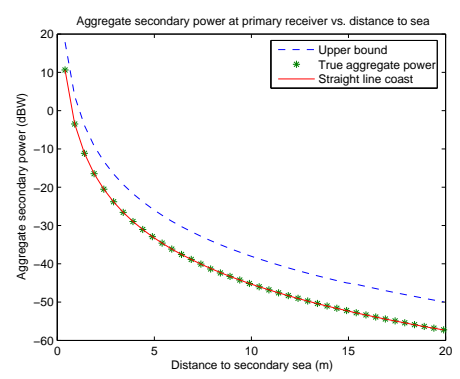

Fig. 6. The upper (eqn. 14) and lower (eqn. 12) bounds have the same decay exponent. Approximating the coast of the secondary sea with a straight line is accurate to within a constant.

\section{MULTIPLE DYNAMIC SECONDARY TRANSMITTERS}

We now consider the case in which secondary users are allowed to increase the power of their transmissions as they move further from the protected region. Clearly, the rule we pick to govern $D(r)$ will determine the impact of the secondary sea on the primary receivers. Noting that $\frac{1}{g_{21}(r)}$ is an increasing function, we assume the power density to be governed by a rule of the form:

$$
D(r)=D_{0}(\rho) \frac{1}{g_{21}(r)} r^{-\rho}
$$

where $\rho$ is a constant that determines how aggressively the power density $D(r)$ should increase with $r$. This rule can be expressed in terms of local SNR, $\psi$, in (20).

To determine the aggregate interference at a primary receiver on the edge of the protected region, we use the straight line approximation for the coast of the sea. For a particular $\rho$ :

$$
\begin{aligned}
Q_{2} & =\int_{-\frac{\pi}{2}}^{\frac{\pi}{2}} \int_{\frac{r_{n}-r_{p}}{\cos (\theta)}}^{\infty} D(r) g_{21}(r) r d r d \theta \\
& =\frac{D_{0}(\rho)}{-\rho+2} \int_{-\frac{\pi}{2}}^{\frac{\pi}{2}}\left[r^{-\rho+2}\right]_{\frac{r_{n}-r_{p}}{\cos \theta}}^{\infty} d \theta
\end{aligned}
$$

If $\rho>2$, i.e. $D\left(r_{2}\right)$ grows sufficiently slower than $g_{21}\left(r_{2}\right)$, then the integral converges.

$$
\begin{aligned}
& =\frac{D_{0}(\rho) \int_{-\frac{\pi}{2}}^{\frac{\pi}{2}}(\cos \theta)^{\rho-2} d \theta}{\rho-2}\left(r_{n}-r_{p}\right)^{-\rho+2} \\
& =K(\rho) \cdot D_{0}(\rho) \cdot\left(r_{n}-r_{p}\right)^{-\rho+2}
\end{aligned}
$$

With this particular rule for the power density, the sea of secondary transmitters behaves like a single transmitter located $r_{n}-r_{p}$ from the protected radius, with power $K(\rho) \cdot D_{0}(\rho)$ 
and gain function $g_{21}(r)=r^{-\rho+2}$. We can plug our expression straight into (8) to get an bound on $D_{0}(\rho)$.

$$
D_{0}(\rho) \leq \sigma^{2} \frac{\left(10^{\frac{\mu}{10}}-1\right)}{K(\rho) \cdot\left(g_{12}^{-1}\left(10^{\frac{-\Delta-\nu}{10}}\right)-g_{11}^{-1}\left(10^{\frac{-\Delta+\mu}{10}}\right)\right)^{-\rho+2}}
$$

From (6) and (7) we can express $r$ in terms of SNR:

$$
r=r_{2}-r_{p}=g_{12}^{-1}\left(10^{\frac{-\Delta-\psi}{10}}\right)-g_{11}^{-1}\left(10^{\frac{-\Delta+\mu}{10}}\right)
$$

With (18) and (19) we can express (15) as a function of $\psi$ :

$$
\begin{aligned}
D(\psi)= & \sigma^{2}\left(\frac{\left(10^{\frac{\mu}{10}}-1\right)}{K(\rho) \cdot\left(g_{12}^{-1}\left(10^{\frac{-\Delta-\nu}{10}}\right)-g_{11}^{-1}\left(10^{\frac{-\Delta+\mu}{10}}\right)\right)^{-\rho+2}}\right) \\
& \left(\frac{\left(g_{12}^{-1}\left(10^{\frac{-\Delta-\psi}{10}}\right)-g_{11}^{-1}\left(10^{\frac{-\Delta+\mu}{10}}\right)\right)^{-\rho}}{g_{21}\left(g_{12}^{-1}\left(10^{\frac{-\Delta-\psi}{10}}\right)-g_{11}^{-1}\left(10^{\frac{-\Delta+\mu}{10}}\right)\right)}\right)
\end{aligned}
$$

This equation gives the the allowable power density for secondary transmitters as a function of distance measured in $\mathrm{dB}$ from the protected region. The primary receivers have a certain margin $\mu$ of tolerable interference that through policy decisions can be allocated to users at different distances. The more aggressively (smaller $\rho$ ) the secondary transmitters increase their power with distance, the quieter the secondary transmitters near the primary system must become.

\section{Minimum Detectable SNR}

In the preceding sections we mentioned an implicit cap on the transmit powers of the secondary users. Many factors contribute to this upper limit on power, including safety or hardware limitations. It is also affected by a radio's sensitivity:

As a secondary transmitter moves away from the protected radius, its allowable power increases exponentially. At some distance $r_{\max }$, however, the local SNR at the secondary transmitter will drop below its minimum detectable SNR, $\gamma_{\min }$ [7]. From this point outwards, the secondary receiver cannot assume it is more than a distance $r_{\max }$ away from the transmitter, no matter what its actual distance. As a result, there is an absolute cap on the secondary transmit power. This in turn changes the aggregate interference at a primary transmitter on the border of the protected region.

$$
\begin{aligned}
Q_{2}= & \int_{-\frac{\pi}{2}}^{\frac{\pi}{2}} \int_{\frac{r_{n}-r_{p}}{\cos (\theta)}}^{r_{\max }} D(r) g_{21}(r) r d r d \theta \\
& +\int_{-\frac{\pi}{2}}^{\frac{\pi}{2}} \int_{r_{\max }}^{\infty} D\left(r_{\max }\right) g_{21}(r) r d r d \theta \\
= & \frac{D_{0}}{-\rho+2} \int_{-\frac{\pi}{2}}^{\frac{\pi}{2}}\left[r^{-\rho+2}\right]_{\frac{r n-r_{p}}{\cos \theta}}^{r_{\max }} d \theta \\
& +\frac{D\left(r_{\max }\right)}{-\rho+2} \int_{-\frac{\pi}{2}}^{\frac{\pi}{2}}\left[r \tilde{g}_{21}(r)-\check{g}_{21}(r)\right]_{r_{\max }}^{\infty} d \theta \\
= & K(\rho) \cdot D_{0} \cdot\left(r_{n}-r_{p}\right)^{-\rho+2}-\frac{D_{0}}{\rho-2} \pi r_{\max }-\rho+2 \\
& +\frac{D\left(r_{\max }\right)}{\rho-2} \pi\left[\check{g}_{21}\left(r_{\max }\right)-r_{\max } \tilde{g}_{21}\left(r_{\max }\right)\right]
\end{aligned}
$$

We can express $r_{\max }$ in terms of $\gamma_{\min }$.

$$
\begin{aligned}
& \gamma_{\min }=10 \log \left(\frac{P_{1} g_{12}\left(r_{\max }\right)}{\sigma^{2}}\right)-\beta \\
& r_{\text {max }}=g_{12}^{-1}\left(\frac{\sigma^{2}}{P_{1}} 10^{\frac{\gamma_{\min }+\beta}{10}}\right)
\end{aligned}
$$

We can use (22), (23), and (4) to find a bound on $D_{0}$. Alternatively, we could solve this expression for $\gamma_{\min }$. If a manufacturer wanted to build cognitive radios that avoid interfering with legacy systems, $\gamma_{\min }$ represents how sensitive his radios' detection hardware must be.

\section{Conclusion}

We have considered the case of a single legacy transmitter whose spectrum is shared with cognitive radios. The cognitive radios must adjust their power levels according to their potential proximity to a protected receiver. The local SNR of the primary signal provides a viable metric for an effective power control rule. Allowing multiple cognitive systems results in slower effective propagation path loss, though not so slow as to preclude the possibility of practical cognitive radio systems. Adequate sensitivity is a fundamental requirement for a successful cognitive radio. Where shadowing is possible, cognitive radios must compensate by detecting lower SNR signals.

\section{ACKNOWLEDGEMENTS}

Many thanks to the anonymous reviewers, whose insightful comments made this a better paper.

\section{REFERENCES}

[1] R. W. Broderson, A. Wolisz, D. Cabric, S. M. Mishra, and D. Willkomm. (2004) White paper: CORVUS: A cognitive radio approach for usage of virtual unlicensed spectrum. [Online]. Available: http: //bwrc.eecs.berkeley.edu/Research/MCMA/CR_White_paper_final1.pdf

[2] Y. Benkler, "Overcoming agoraphobia: Building the commons of the digitally networked environment," Harvard Journal of Law \& Technology, vol. 11, pp. 287-400, Winter 1998.

[3] New America, et al., "Technical reply," Jan. 2005. [Online]. Available: http://www.newamerica.net/Download_Docs/pdfs/Doc_File_2202_1.pdf

[4] I. J. Mitola, "Software radios: Survey, critical evaluation and future directions," IEEE Aerosp. Electron. Syst. Mag, vol. 8, pp. 25-36, Apr. 1993.

[5] FCC, "FCC 03-322," Dec. 2003. [Online]. Available: http://hraunfoss. fcc.gov/edocs_public/attachmatch/FCC-03-322A1.pdf

[6] A. Sahai, N. Hoven, and R. Tandra, "Some fundamental limits on cognitive radio," in Forty-second Allerton Conference on Communication, Control, and Computing, Monticello, IL, Oct. 2004.

[7] R. Tandra and A. Sahai, "Fundamental limits on detection in low SNR under noise uncertainty," in WirelessCom 2005, Maui, HI, June 13-16, 2005.

[8] W. Lee, Mobile communication design fundamentals, 2nd ed.

[9] - Mobile cellular telecommunications, 2nd ed.

[10] T. S. Rappaport and L. B. Milstein, "Effects of radio propagation path loss on DS-CDMA cellular frequency reuse efficiency for the reverse channel," IEEE Trans. Veh. Technol., vol. 41, pp. 231-242, Aug. 1992.

[11] X. Guo, S. Roy, and W. S. Connor, "Spatial reuse in wireless ad-hoc networks," in Proc. 2003 IEEE Veh. Technol. Conf., vol. 3, Oct. 6-9, 2003, pp. 1437-1442.

[12] J. Zhu, X. Guo, L. L. Yang, and W. S. Conner, "Leveraging spatial reuse in 802.11 mesh networks with enhanced physical carrier sensing," in Proc. 2004 IEEE Int. Commun. Conf., vol. 7, June20-24 2004, pp. 4004-4011. 
[13] D. Tse and P. Viswanath, Fundamentals of Wireless Communication, 2005. [Online]. Available: http://www.ifp.uiuc.edu/ $\sim$ pramodv/pubs/ book090904.pdf

[14] FCC, "FCC 04-113," May 2004. [Online]. Available: http://hraunfoss. fcc.gov/edocs_public/attachmatch/FCC-04-113A1.pdf

[15] J. A. Fuemmeler, N. H. Vaidya, and V. V. Veeravalli, "Selecting transmit powers and carrier sense thresholds for CSMA protocols," University of Illinois at Urbana-Champaign, IL, Tech. Rep., Oct. 2004.

[16] Q. T. Zhang, "Bridging the gap between dynamic and static methods for cell planning," IEEE Trans. Veh. Technol., vol. 50, pp. 1224-1230, Sept. 2001. 